\title{
2D Discrete Mirror Transform for Image Non-Linear Approximation
}

\author{
Alessandro Gnutti, Fabrizio Guerrini, and Riccardo Leonardi \\ Department of Information Engineering, CNIT - University of Brescia, Italy \\ Email: name.surname@unibs.it
}

\begin{abstract}
In this paper, a new 2D transform named Discrete Mirror Transform (DMT) is presented. The DMT is computed by decomposing a signal into its even and odd parts around an optimal location in a given direction so that the signal energy is maximally split between the two components. After minimizing the information required to regenerate the original signal by removing redundant structures, the process is iterated leading the signal energy to distribute into a continuously smaller set of coefficients. The DMT can be displayed as a binary tree, where each node represents the single (even or odd) signal derived from the decomposition in the previous level. An optimized version of the DMT (ODMT) is also introduced, by exploiting the possibility to choose different directions at which performing the decomposition. Experimental simulations have been carried out in order to test the sparsity properties of the DMT and ODMT when applied on images: referring to both transforms, the results show a superior performance with respect to the popular Discrete Cosine Transform (DCT) and Discrete Wavelet Transform (DWT) in terms of non-linear approximation.
\end{abstract}

\section{INTRODUCTION}

Linear transformations are largely used in many signal processing applications, such as signal approximation, data compression, denoising, and so on [1]. In general, an efficient transform should be able to closely approximate the signal by using a small number of transform coefficients, that is, efficiently-performing transforms should generate a sparse representation of a signal. The search for a universal optimal transform is a very arduous problem, since typically it has to satisfy very different task constraints and fit in with distinct classes of signals [2].

Two families of transforms can be categorized in terms of design techniques. The first category assumes an a priori knowledge of a good statistical model. Typically, this family of transforms is well suited when regular behaviors are present within the data samples, e.g., piece-wise smoothness. Then, these model-based approaches attempt to describe signal variations with a few transform coefficients. Popular transforms belonging to this category are the Fourier transform, the cosine transform, wavelets, and the more recent Curvelets [3], Contourlets [4], Bandelets [5] or Directionlets [6], in addition to the graph Fourier transforms [7][8] that exploit the structure of some graphs in order to model the signal, e.g., [9] (actually, in some works the graph is constructed by learning graph Laplacians so that a particular class of input data forms graph signals with smooth variations on the resulting topology [10]; this case belongs to the second category).
The second class of design techniques tries to capture more general forms of regularity that may be hard to describe with concise analytical models. Some data-driven algorithms can be used to seek transforms exhibiting good linear or non-linear approximation capabilities. For example, principal component analysis (PCA) and the Karhunen-Loeve transform (KLT), that exploit the correlation of the data, are proved to be optimal in terms of linear approximation. However, when compression and signal representation have shifted from PCA and linear approximation to non-linear approximation, other methods caught on. Typically, these new techniques impose some algebraic restrictions on the transform coefficients, in order to achieve a prefixed level of sparsity. Emblematic examples are the KSVD (and, in general, algorithms that create over-complete dictionaries) [11], the Sparse Orthonormal Transform (that tries to generalize the KLT) [2], in addition to design techniques using various optimization methods.

In this paper, we present a new multidimensional transform, named Discrete Mirror Transform (DMT). The framework is inspired by what was proposed in [12], evolving the ideas expressed therein, and in [13], of which this work provides a natural extension into the $2 \mathrm{D}$ domain. Such a transform can be associated to the aforementioned second category, even if with an important difference. Indeed, the representation of the signal does not derive from a projection on the space spanned by a predefined set of vectors. On the contrary, each signal is expressed through an iterative optimal even/odd decomposition with respect to a given direction that distributes the energy of the signal into a smaller number of coefficients at each iteration. The discrete mirror transform can be visualized as a binary tree, where a node in a certain level represents a single decomposition (even or odd) referred to the parent node at the previous level, and the leaf nodes determine the final transform coefficients. The process is invertible, that is, the original signal can be reconstructed given the transform coefficients and the location of the leaf nodes in the binary tree.

Since the mirroring location is calculated so as to provide maximal energy concentration into the even or odd part, the coefficients will be characterized by a strong sparsity that allows to well approximate the original signal by keeping just a limited set of them. In other words, the architecture of the algorithm that builds the DMT naturally defines a transform that compacts the signal energy into a few of its components. As a matter of fact, in this work the efficiency of the DMT is tested on images: the results show performance higher 
with respect to other popular transforms when a non-linear approximation is applied in the transform domain.

An important property of the DMT is that it preserves the energy of the signal, differently from some recent works providing non-orthogonal and overcomplete transforms. Nonorthogonality leads to involved search techniques even for simple operations, e.g., scalar quantization. Our transform, by preserving the validity of the Parseval's theorem beyond the linear expansion context, is exempt from such issues. Moreover, as it will be described in detail in the next sections, the energy preservation holds not only in the transform domain, but even for each level of the binary tree. It means that zeroing (or quantizing) an intermediate node of the tree has the same effect of a non-linear approximation (or quantization) of the transform coefficients, even leading to a more compact information to locate the non-zero coefficients. Also in this case, the DMT preserves its invertibility, as long as the interruption of the iteration at that specific node is signalled within the binary tree.

The rest of the paper is organized as follows. In Sec. II, how to find the optimal symmetry point/plane in a $2 \mathrm{D}$ signal is described. Sec. III defines the analysis and synthesis operations of the discrete mirror transform and its representation as a decomposition binary tree. In Sec. IV some experiments are carried out to show the sparsity property of the DMT and to compare its non-linear approximation ability with respect to typical linear expansions. Finally, some conclusions are drawn in Sec. V.

\section{2D OPTIMAL SYMMETRY DECOMPOSITION}

Given a $l^{2}$-norm signal $x[m]: \mathbb{Z} \rightarrow \mathbb{R}$, it can be always expressed as the sum of its even and odd part, say $x_{e}[m]$ and $x_{o}[m]$, respectively. Generally, when inspecting the evenness and oddness of a signal, the line $m=0$ is implicitly considered as the symmetry axis. However, it is possible to define an extended notion of symmetry by decomposing $x[m]$ around an arbitrary point $m_{0}$ as follows.

$$
\begin{aligned}
& x_{e}\left[m ; m_{0}\right]=\frac{x[m]+x\left[2 m_{0}-m\right]}{2} \\
& x_{o}\left[m ; m_{0}\right]=\frac{x[m]-x\left[2 m_{0}-m\right]}{2} .
\end{aligned}
$$

In [14] the authors showed how to detect the global optimal symmetry point $m_{0}^{\prime}$, i.e., the point around which the even/odd decomposition maximally splits the energies of the even and odd components. It turns out that the optimal location is strictly related to the self-convolution of the signal, in particular:

$$
2 m_{0}^{\prime}=\underset{k}{\arg \max }|(x * x)[k]| .
$$

The same study can be clearly extended in multidimensional spaces. Focusing on the 2D case, i.e., considering the $l^{2}$-norm signal $x[m, n]: \mathbb{Z}^{2} \rightarrow \mathbb{R}^{2}$, the even/odd decomposition around a generic point $\left[m_{0}, n_{0}\right]$ is defined as follows:

$$
\begin{aligned}
x_{e}\left[m, n ; m_{0}, n_{0}\right] & =\frac{x[m, n]+x\left[2 m_{0}-m, 2 n_{0}-n\right]}{2} \\
x_{o}\left[m, n ; m_{0}, n_{0}\right] & =\frac{x[m, n]-x\left[2 m_{0}-m, 2 n_{0}-n\right]}{2} .
\end{aligned}
$$

Specifically, Eq. (3) refers to a so-called point reflection.

In order to find the global optimal symmetry point $\left[m_{0}^{\prime}, n_{0}^{\prime}\right]$, the procedure described in [14] for the 1D domain can be properly adjusted for the $2 \mathrm{D}$ domain. Let $E_{e}\left[m_{0}, n_{0}\right]$ and $E_{o}\left[m_{0}, n_{0}\right]$ be the functions of the energies associated to $x_{e}$ and $x_{o}$, respectively, as the point $\left[m_{0}, n_{0}\right]$ varies. The expression of the even energy function can be written as:

$$
\begin{aligned}
E_{e}\left[m_{0}, n_{0}\right] & =\sum_{\mathbb{Z}^{2}}\left|x_{e}\right|^{2}=\sum_{\mathbb{Z}^{2}}\left|\frac{x+x_{m_{0}, n_{0}}}{2}\right|^{2}= \\
& =\frac{1}{4} \sum_{\mathbb{Z}^{2}}\left|x^{2}+x_{m_{0}, n_{0}}^{2}+2 x \cdot x_{m_{0}, n_{0}}\right|
\end{aligned}
$$

where, for the sake of conciseness, $x$ and $x_{m_{0}, n_{0}}$ are used in place of $x[m, n]$ and $x\left[2 m_{0}-m, 2 n_{0}-n\right]$, respectively. The first two terms in the last summation return the energy of the signal, say $E$, since the reversing and shifting operations do not affect the energy value of a signal (note that $E=E_{e}+E_{0}$, since $x_{e} \perp x_{o}$ ). Then:

$$
E_{e}\left[m_{0}, n_{0}\right]=\frac{1}{2} E+\frac{1}{2} \underbrace{\sum_{\mathbb{Z}^{2}} x[m, n] \cdot x\left[2 m_{0}-m, 2 n_{0}-n\right]}_{(:=x * * x)\left[2 m_{0}, 2 n_{0}\right]}
$$

where the last term is exactly the $2 \mathrm{D}$ discrete linear convolution denoted by $* *$. By repeating the same process for $E_{o}$ as well, we obtain:

$$
\begin{aligned}
& E_{e}\left[m_{0}, n_{0}\right]=\frac{1}{2} E+\frac{1}{2}(x * * x)\left[2 m_{0}, 2 n_{0}\right] \\
& E_{o}\left[m_{0}, n_{0}\right]=\frac{1}{2} E-\frac{1}{2}(x * * x)\left[2 m_{0}, 2 n_{0}\right] .
\end{aligned}
$$

It turns out that the energy functions $E_{e}$ and $E_{o}$ depend on the behavior of the $2 \mathrm{D}$ self-convolution of the signal. Then, determining the optimal $\left[m_{0}, n_{0}\right]$ point that maximally splits the energy values is computed by:

$$
\left[2 m_{0}^{\prime}, 2 n_{0}^{\prime}\right]=\underset{(k, h)}{\arg \max }|(x * * x)[k, h]| .
$$

Note that $n_{0}^{\prime}$ and $m_{0}^{\prime}$ can exclusively be integers or halfintegers. Instead of dealing with both cases we simply choose the coordinates to be just half-integers without affecting the general flow of the presentation, i.e., whenever $n_{0}^{\prime}$ and $m_{0}^{\prime}$ turn out to be integers, then \pm 0.5 is added based on the corresponding largest convolution values.

Generally, symmetries with respect to a plane in 2D are also of interest. For example, by considering $n=n_{0}$ as the 


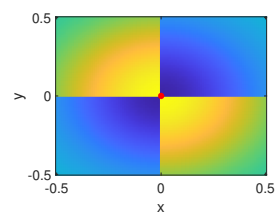

(a) Evenness w.r.t. the $[0,0]$ point.

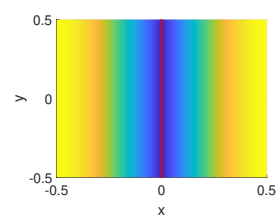

(b) Evenness w.r.t. the plane $n=0$.

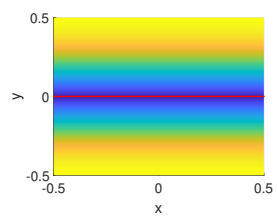

(c) Evenness w.r.t. the plane $m=0$.
Fig. 1: Illustration of (even) symmetries with respect to the $[0,0]$ point, the plane $n=0$ and the plane $m=0$. The function values are represented in pseudocolor. The symmetry point and planes are drawn in red.

vertical reflection plane, the even/odd components of $x[m, n]$ with respect to it are expressed as:

$$
\begin{aligned}
x_{e}\left[m, n ; n_{0}\right] & =\frac{x[m, n]+x\left[m, 2 n_{0}-n\right]}{2} \\
x_{o}\left[m, n ; n_{0}\right] & =\frac{x[m, n]-x\left[m, 2 n_{0}-n\right]}{2} .
\end{aligned}
$$

Thus, repeating the steps previously reported for the point reflection, the new energy function $E_{e}\left[n_{0}\right]$ is equal to:

$$
E_{e}\left[n_{0}\right]=\frac{1}{2} E+\frac{1}{2} \underbrace{\sum_{\mathbb{Z}^{2}} x[m, n] \cdot x\left[m, 2 n_{0}-n\right]}_{(x * x)\left[2 n_{0}\right]}
$$

where the last term is exactly the 1D discrete linear convolution applied to a 2D signal. In this case, Eq. (7) becomes:

$$
2 n_{0}^{\prime}=\underset{h}{\arg \max }|(x * x)[h]| .
$$

Obviously, when considering the reflection with respect to $m=$ $m_{0}$, the same result is obtained by just applying the mirroring in the orthogonal direction, namely using an horizontal plane.

As an illustrative example, a point reflection and two symmetries with respect to the plane $n=0$ and the plane $m=0$ are shown in Fig. 1a, Fig. 1b and Fig. 1c, respectively. For the sake of clarity, let us consider the first case: following the definition reported in Eq. 7, the global optimal symmetry point of the function $x[m, n]$ depicted in Fig. 1a is clearly $\left[x_{0}, y_{0}\right]=[0,0]$. Considering the symmetry around this point, $x_{e}[m, n]=x[m, n]$ and $x_{o}[m, n]=0$ (Eq. 3), that is, in the even/odd decomposition the energy of the signal is completely distributed in its even part (this, of course, represents the ideal case where the examined function is purely even (odd) and thus its energy is maximally split).

\section{2D DISCRETE MIRROR TRANSFORM}

\section{A. One-step iteration}

In this section, we introduce the connection between optimal even/odd decomposition and sparsity, by concentrating the analysis on images data. Note that the types of symmetry as defined in Sec. II for a generic 2D discrete signal can be thought as symmetries with respect to a single pixel or a row/column. For the sake of conciseness, this section will treat

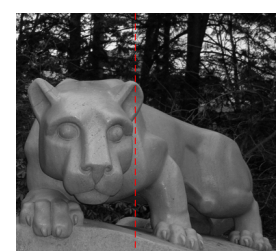

(a) Reference image.

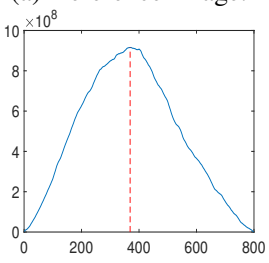

(d) Conv. - Eq. (10)

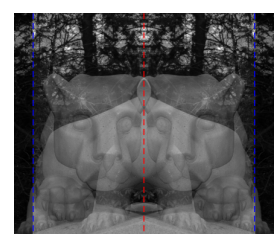

(b) Even part - Eq. (8).

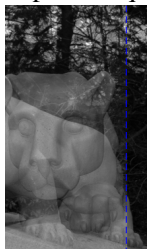

(e) Causa even part (with tail).

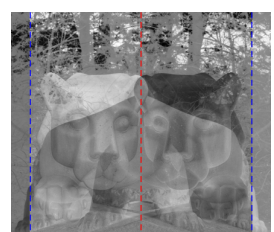

(c) Odd part - Eq. (8)
Fig. 2: Even/odd decomposition ( $2 b$ and $2 c$ ) of the image (2a) with respect to the optimal symmetry column found by identifying the maximum of the convolution (2d). The minimal information required to reconstruct the original signal are depicted in $2 \mathrm{e}$ and $2 \mathrm{f}$.

just symmetry with respect to a column (the same approach can be applied exactly in the same way for symmetry around a pixel or with respect to a row).

Let $I[m, n]$ be an image defined in $[0, M-1] \times[0, N-1]$. In order to find the global optimal symmetry column, we need to compute the maximum of the corresponding self-convolution modulus as described in Eq. (10). As an example, Fig. 2d depicts the self-convolution modulus associated to the image shown in Fig. 2a: the location of the maximum, say $i_{M}$, is indicated with a red dashed line as well as the matching optimal symmetry column $n_{0}^{\prime}=\frac{i_{M}}{2}$ in the image. In order to compute $I_{e}[m, n]$ and $I_{o}[m, n]$, the even/odd decomposition around $n_{0}=n_{0}^{\prime}$ is applied to $I[m, n]$ as in Eq. (8): Figs. $2 \mathrm{~b}$ and $2 \mathrm{c}$ show the even and odd part, respectively. The number of columns of the two images increases to $N+2 t$, where $t$ is the absolute value of the distance between $n_{0}^{\prime}$ and the middle column $\frac{N}{2}$. However, note that both include a $2 t$ long support that represents the "tail" of the original image: such regions are identified by the blue dashed lines in Figs. 2b and 2c.

At this point, it may be wondered how to regenerate the original image by minimizing as much as possible the information contained into the even and odd components. To answer this question, the first observation is that both $I_{e}[m, n]$ and $I_{o}[m, n]$ are completely defined by the last (first) half of the columns, since the other half can be obtained by an even/odd mirroring operation (let us call $I_{e c}$ and $I_{o c}$ the "causal" parts of $I_{e}$ and $I_{o}$ ). A second consideration is that the tail can be removed either in $I_{e c}$ or $I_{o c}$ without losing any information, since it is shared between both the signals. Then, by removing the tail, for example, in $I_{o c}$, the minimal information of the even/odd decomposition necessary to reconstruct the original image is finally achieved (Figs. 2e and 2f). Note that the sum of the columns of the two final images is exactly equal to $N$. 


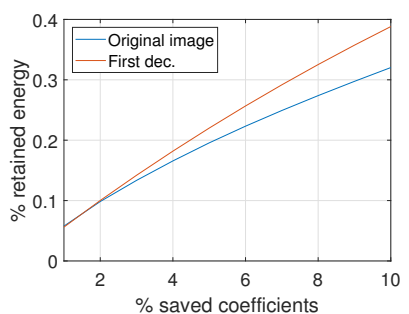

(a) Measure of the energy compaction before and after the decomposition.

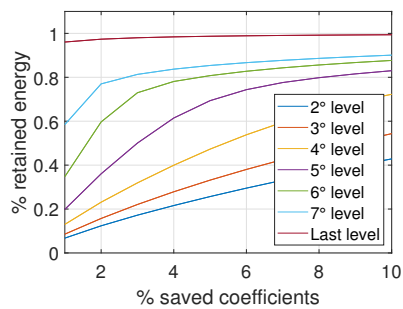

(b) Measure of the energy compaction at varying of the decomposition level.
Fig. 3: Evaluation of the sparsity of the resulting signal when iterating the optimal symmetry decomposition.

The operations to regenerate the original signal are straightforward: first, the causal even part without the tail and the causal odd part are extended by the appropriate mirroring operation. Then, the resulting signals are added and, lastly, the tail is concatenated.

\section{B. Binary Tree: Signal Decomposition}

Let us denote with $I_{\mathcal{E}}$ and $I_{\mathcal{O}}$ the signals shown in Figs. 2e and $2 \mathrm{f}$, respectively. As mentioned before, these two signals allow to reconstruct the original image. The improvement of this representation is that a certain quantity of energy is moved from the image pixels to a smaller set of samples. In our example, we have $E_{e}\left[n_{0}^{\prime}\right] / E=0.86$.

Fig. 3a reports two curves: the blue one represents the ratio between the energy of $I$ when keeping just a certain percentage of the largest pixel values (in modulus) and the total energy of $I$. The red curve evaluates the same ratio, but considering the "concatenation" of $I_{\mathcal{E}}$ and $I_{\mathcal{O}}$ instead of the original image. Interestingly, the second curve overcomes the first one, meaning that the energy of the concatenation of $I_{\mathcal{E}}$ and $I_{\mathcal{O}}$ is more concentrated in a few samples with respect to how much it is in $I$.

Starting from this preliminary observation, we propose to iterate the even/odd decomposition up to when single coefficients are achieved. In this way, a binary tree, whose nodes represent the even or the odd part obtained from a previous decomposition, is generated. We call this procedure the discrete mirror transform (DMT) of a signal. Basically, the two signals $I_{\mathcal{E}}$ and $I_{\mathcal{O}}$ constitute the first-level nodes of the tree. For the sake of clarity, let us rename them $I_{\mathcal{E}}^{(1)}$ and $I_{\mathcal{O}}^{(1)}$. Then, the even/odd decomposition is distinctly applied to $I_{\mathcal{E}}^{(1)}$ and $I_{\mathcal{O}}^{(1)}$ again, generating the four nodes $I_{\mathcal{E} \mathcal{E}}^{(2)}, I_{\mathcal{E} \mathcal{O}}^{(2)}$, $I_{\mathcal{O} \mathcal{E}}^{(2)}$ and $I_{\mathcal{O O}}^{(2)}$. In general, a node at level $l$ is referred to as $I_{\mathcal{S}_{1} \mathcal{S}_{2} \ldots \mathcal{S}_{l}}^{(l)}$, where $S_{i}$ represents the symbol $\mathcal{E}$ or $\mathcal{O}$, depending on from which part of the decomposition it derives (even or odd).

Two final considerations have to be stated at this point. The first one is that each branch ends with a leaf node containing a single coefficient. Indeed, when a node contains a single coefficient the iteration for that node clearly stops, since computing a further even/odd decomposition is useless

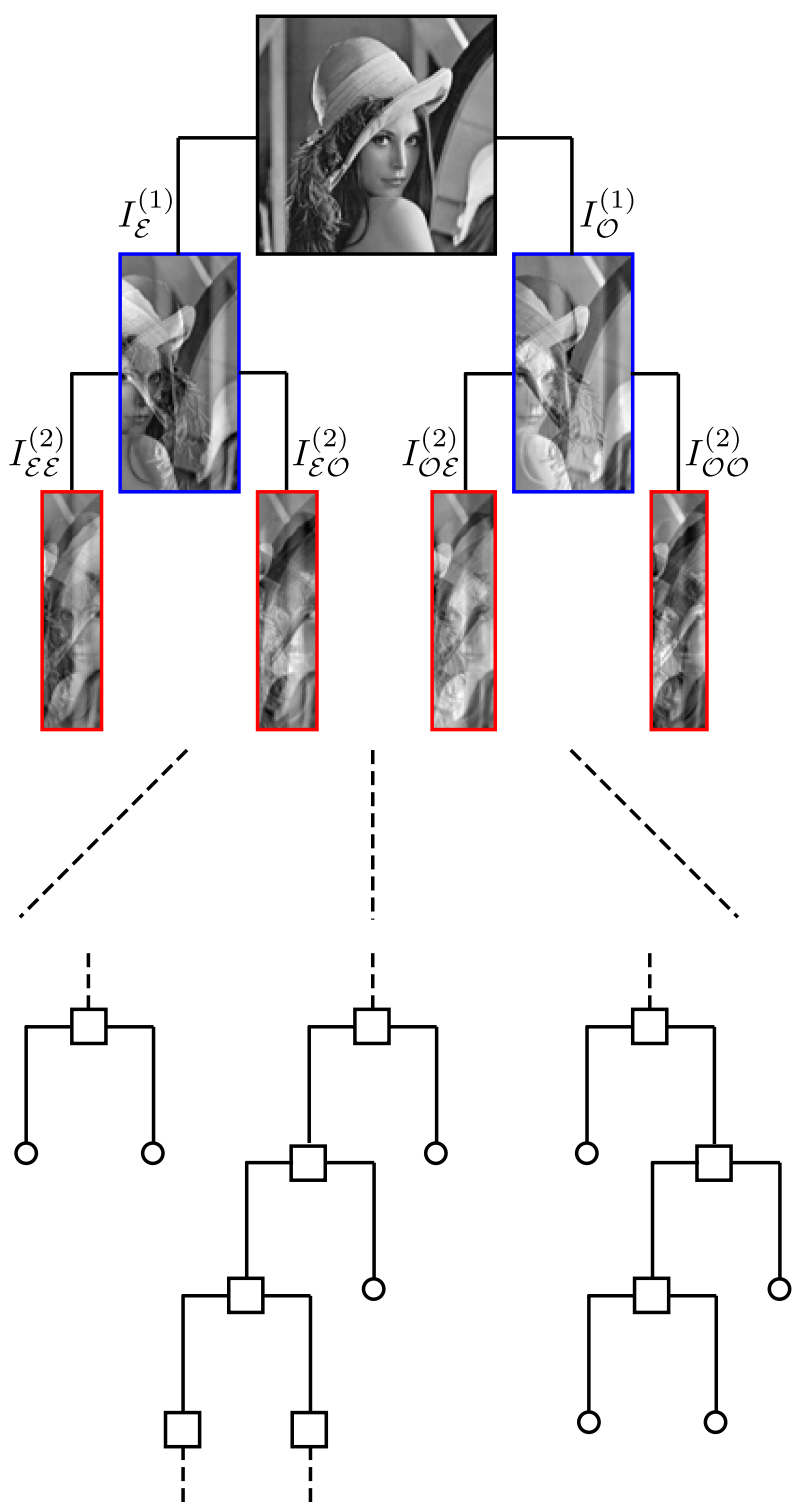

Fig. 4: A part of the binary tree corresponding to the Lena image decomposition. The first two levels are entirely shown, while some other nodes are depicted for deeper levels when zooming in a small region of the tree. Here, the squares indicate generic nodes from which the decomposition keeps on, while the circles represent the leaves, namely the transform coefficients. Note that the coefficients are generally located at different levels of the tree.

(note that when a node is composed by just a column, the analysis simply move in the 1D domain without varying the decomposition process). The important fact is that the single coefficients can be located at different levels, since the size of the nodes tends to unequally decrease while descending the tree. This evidence leads to the conclusion that the binary tree is not balanced, i.e., the leaf nodes are located at different levels. As an example, some portions of the binary tree associated to the Lena image decomposition are illustrated in 
Fig. 4.

The second observation is that by taking all the nodes belonging to a specific level, the energy of the original signal is preserved. What it changes is the distribution of the energy over the samples. Fig. $3 \mathrm{~b}$ presents the curves related to the energy functions when varying the number of the kept largest coefficients (in modulus) for each level (note that the curve labeled as last level refers to the final coefficients of the transform). What emerges is that the higher is the level, i.e., the deeper is the decomposition, the sparser is the signal representation at that corresponding level, proving the strong sparsity property of the discrete mirror transform.

\section{Binary Tree: Signal Reconstruction}

In Sec. III-A, we mentioned the fact that given two nodes, the parent node of the previous level can be obtained by 1) removing the tail from the even node, 2) extending the causal even and odd parts with respect to the corresponding mirroring operation, 3) summing the obtained signals, and 4) concatenating the tail with the resulting signal.

However, we have assumed to know the point that separates the causal even part and the tail. Therefore, one may wonder if the optimal symmetry position $n_{0}^{\prime}$ is needed for the reconstruction process. Actually, the number of columns of the two nodes identify the unique two allowable locations around which the even/odd decomposition may have been applied. Let us call $L_{e}$ and $L_{o}$ the number of columns of the even and odd node, respectively, with $L_{e} \geq L_{o}$ since the even node contains the tail as well. The parent node will have $L_{e}+L_{o}$ columns, and the optimal symmetry column could be both $n_{0}^{\prime}=L_{o}$, when $n_{0}^{\prime}$ is in the first half of the columns, and $n_{0}^{\prime}=L_{e}$, otherwise (since a signal and its flipped version would return the same decomposition).

In order to overcome this potential ambiguity, it is sufficient to save the tail of the even part when $n_{0}^{\prime}$ is to the left of the support mid-column, otherwise saving the tail of the odd part. Note that in this way $L_{o}$ consistently returns the optimal symmetry position.

One last observation is that the single coefficients are sufficient to regenerate the original signal, that is, the signals in the intermediate nodes are not necessary. Indeed, by computing the reconstruction process from the leaf nodes, the rest of the tree is automatically generated. Actually, what the inverse algorithm needs is the position of the leaf nodes. It means that the structure of the tree has to be known, i.e., each node of the tree has to indicate if the iteration has continued or if a single coefficient has been reached.

\section{EXPERIMENTAL RESULTS}

In this section, some experiments aimed to highlight the sparsity properties of the discrete mirror transform are presented. They have been tested on various databases including different types of image, such as miscellaneous (41 images), aerial (30 images) and textures (64 images) for standard definition images taken from [15], and 15 high definition images taken from [16].
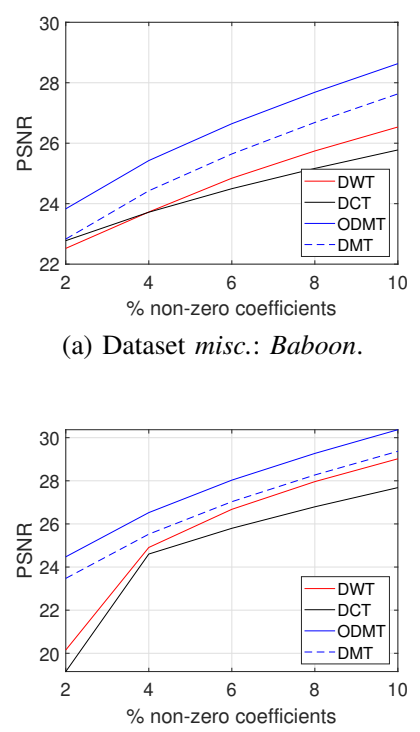

(c) Dataset aerial: 5.1.14.

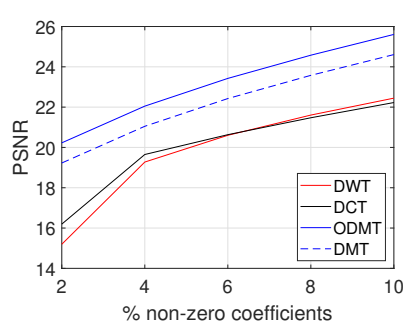

(e) Dataset textures: 1.1.02.

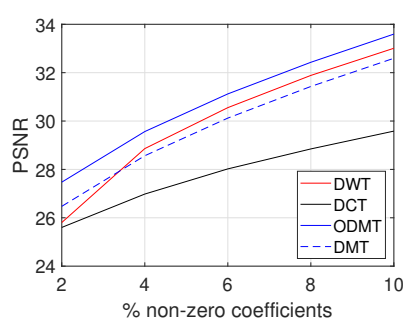

(g) HD dataset: Cathedral. (a) Dataset misc.: Baboon.

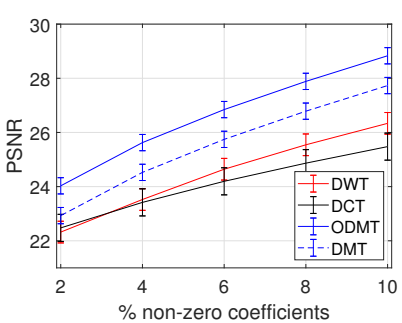

(b) Dataset misc.: average and standard deviation.

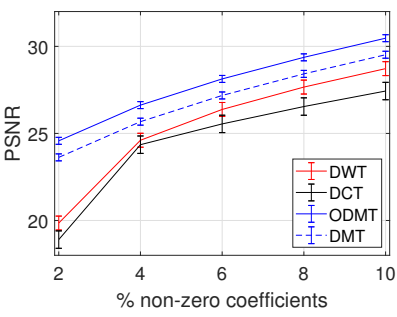

(d) Dataset aerial: average and standard deviation.

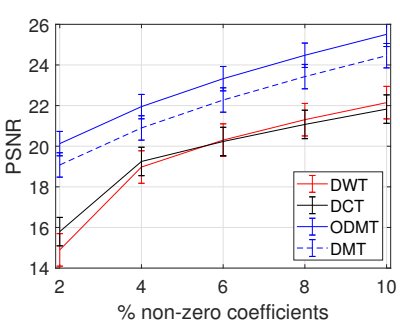

(f) Dataset textures: average and standard deviation.

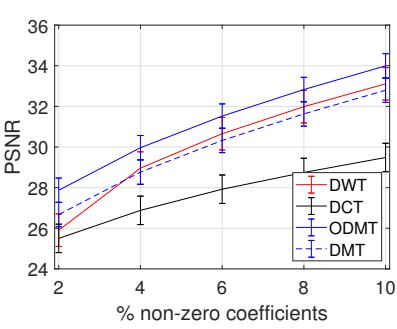

(h) HD dataset: average and standard deviation.
Fig. 5: Performance comparison between DCT, DWT, DMT and ODMT when applied to some images of the considered datasets (left column). The average performance is also shown in the right column.

To measure the ability of the discrete mirror transform to compact the energy to a small set of coefficients, it has been compared with other two popular transforms: the Discrete Cosine Transform (DCT) and the Discrete Wavelet Transform (DWT) implemented using the 'db4' wavelet with 4 decomposition levels. The comparison has been performed by applying a non-linear approximation to each transform. Specifically, for the discrete mirror transform the whole decomposition tree is generated and then the original signal is approximated by computing the inverse transform while using just a certain 
percentage of the most significant leaf nodes (in terms of energy). Finally, the PSNR of the reconstructed signal is determined. The same process is performed for the DCT and the DWT, by retaining the most significant coefficients in the frequency or time-frequency domain based on their magnitude.

Fig. 5 depicts the curves of the performance comparison, by emphasizing the result on a single image for each dataset and the average performance with the associated standard deviation as well. Note that a fourth component has been added in addition to the DCT, DWT and DMT. Indeed, an optimized version of the DMT has been implemented as well, and we refer to it as ODMT. The ODMT differs with respect to the DMT based on the fact that for each symmetry decomposition the system decides in which direction to perform the decomposition itself. Specifically, the choice is made between two potential signal decompositions: the first corresponds to the optimal symmetry column (as done for the DMT), while the second considers the orthogonal direction, i.e., the optimal symmetry row. In other words, for each node the optimal decomposition is computed in both directions, and then just the one that returns the maximal separation of the energies is kept.

The experiments show that the DCT and DWT performance are consistently worse than those given by both DMT and ODMT, independently the image class. As expected, the ODMT outperforms the DMT, even if in the optimized version the tree nodes (leaf nodes excepted) also need to include an indication of which direction the decomposition has been performed during the analysis phase.

\section{CONCLUSIONS}

In this paper, the 2D Discrete Mirror Transform (DMT) has been presented, with the objective of finding an efficient transform able to non-linearly approximate images data. The DMT provides for an iterative optimal even/odd decomposition that moves the energy signal into a smaller set of components step by step. A binary tree can be suitably used to represent the DMT: for each iteration a new level of the tree is generated, whose nodes are the (even or odd) decomposition derived from the previous iteration/level. The process ends when a single coefficient is reached. An optimized version of the DMT, named ODMT, has also been presented: it allows to select the optimal symmetry plane between two different orthogonal directions. The experiments have shown high performance by inspecting the sparsity in the transform domain: the reported results have indicated that both the DMT and ODMT outperform the popular DCT and DWT, proving superior capability in terms of images non-linear approximation. Future works will focus on finding an efficient representation of the decomposition binary tree.

\section{REFERENCES}

[1] V. K. Goyal, "Theoretical foundations of transform coding," IEEE Signal Processing Magazine, vol. 18, no. 5, pp. 9-21, Sep. 2001.

[2] O. G. Sezer, O. G. Guleryuz, and Y. Altunbasak, "Approximation and compression with sparse orthonormal transforms," IEEE Transactions on Image Processing, vol. 24, no. 8, pp. 2328-2343, Aug. 2015.
[3] E. Candès and D. D., "Curvelets - a surprisingly effective nonadaptive representation for objects with edges," Curves and Surfaces, April 2000.

[4] M. N. Do and M. Vetterli, "The contourlet transform: an efficient directional multiresolution image representation," IEEE Transactions on Image Processing, vol. 14, no. 12, pp. 2091-2106, Dec. 2005.

[5] E. L. Pennec and S. Mallat, "Sparse geometric image representations with bandelets," IEEE Transactions on Image Processing, vol. 14, no. 4, pp. 423-438, April 2005.

[6] V. Velisavljevic, B. Beferull-Lozano, M. Vetterli, and P. L. Dragotti, "Directionlets: anisotropic multidirectional representation with separable filtering," IEEE Transactions on Image Processing, vol. 15, no. 7, pp. 1916-1933, July 2006.

[7] D. I. Shuman, S. K. Narang, P. Frossard, A. Ortega, and P. Vandergheynst, "The emerging field of signal processing on graphs: Extending high-dimensional data analysis to networks and other irregular domains," IEEE Signal Processing Magazine, vol. 30, no. 3, pp. 83-98, May 2013.

[8] A. Ortega, P. Frossard, J. Kovačević, J. M. F. Moura, and P. Vandergheynst, "Graph signal processing: Overview, challenges, and applications," Proceedings of the IEEE, vol. 106, no. 5, pp. 808-828, May 2018.

[9] A. Gnutti, F. Guerrini, R. Leonardi, and A. Ortega, "Coding of image intra prediction residuals using symmetric graphs," in IEEE International Conference on Image Processing (ICIP), 2019, pp. 131-135.

[10] H. E. Egilmez, E. Pavez, and A. Ortega, "Graph learning from data under laplacian and structural constraints," IEEE Journal of Selected Topics in Signal Processing, vol. 11, no. 6, pp. 825-841, 2017.

[11] M. Aharon, M. Elad, and A. Bruckstein, "K-SVD: An algorithm for designing overcomplete dictionaries for sparse representation," IEEE Transactions on Signal Processing, vol. 54, no. 11, pp. 4311-4322, Nov. 2006.

[12] F. Guerrini, A. Gnutti, and R. Leonardi, "Even/odd decomposition made sparse," Signal Processing, vol. 141, no. C, pp. 273-286, Dec. 2017.

[13] F. Guerrini, A. Gnutti, and R. Leonardi, "Iterative mirror decomposition for signal representation," in IEEE International Conference on Acoustics, Speech and Signal Processing (ICASSP), May 2019, pp. 5541-5545.

[14] A. Gnutti, F. Guerrini, and R. Leonardi, "Representation of signals by local symmetry decomposition," in European Signal Processing Conference (EUSIPCO), Aug. 2015, pp. 983-987.

[15] "The usc-sipi image database," http://sipi.usc.edu/database, 2020, [Online; accessed 15-July-2020].

[16] "Image compression set," http://imagecompression.info, 2020, [Online; accessed 15-July-2020]. 